Multiples Myelom

\title{
Kontinuierliche Behandlung mit Lenalidomid zeigt gute Wirkung
}

Das multiple Myelom (MM) ist in der Regel nicht heilbar. Deutliche prognostische Verbesserungen konnten aber beispielsweise mit Lenalidomid (Revlimid ${ }^{\oplus}$ ) erreicht werden. So reduziert die kontinuierliche Gabe des immunmodulierenden Wirkstoffs (IMiD ${ }^{\oplus}$ ) im Anschluss an eine First-Line-Therapie mit Melphalan - ob konventionell dosiert oder hoch dosiert mit autologer Stammzelltransplantation (ASCT) - das Risiko von Progression oder Tod signifikant.

Interessant sind auch die Ergebnisse der Studie MM-015: In dieser Studie wurde Lenalidomid, das im Gegensatz zu Thalidomid keine relevante Neurotoxizität aufweist, als kontinuierliche Therapie bei neu diagnostizierten MM-Patienten $\geq 65$ Jahren untersucht. In der doppelblinden
Phase-III-Studie wurden 459 Patienten in drei Armen randomisiert behandelt. Zwei Gruppen erhielten jeweils neun Zyklen einer Kombination aus Melphalan, Prednison und Lenalidomid (10 $\mathrm{mg} /$ Tag, Tage 1-21), an die sich entweder eine Behandlung mit Placebo (MPR, $n=153$ ) oder eine kontinuierliche Therapie mit Lenalidomid anschloss (MPR-R, $\mathrm{n}=152$ ). Die dritte Gruppe wurde mit neun Zyklen MP gefolgt von Placebo behandelt. Die Therapie wurde in allen Armen bis zur Progression fortgesetzt. Das Ergebnis: Die Ansprechraten waren im MPR-R-Arm signifikant höher (77 vs. $50 \%$; p <0,001) und die Patienten sprachen schneller auf die Therapie an (2 vs. 3 Monate, $\mathrm{p}<0,001)$. Nach zwei Jahren lebten noch $55 \%$ der mit MPR-R behandelten Patienten zum Teil sogar progressionsfrei (vs. $16 \%$ in der MP-Gruppe). Dies entspricht einer Risikoreduktion für Progression oder Tod von $58 \%$ (HR: $0,423 ; \mathrm{p}<0,001)$.

$\mathrm{db}$

Nach Information von Celgene

\section{Chronisch myeloische Leukämie Nilotinib in der First-Line zugelassen}

\section{Die Europäische Arzneimittelbehörde (EMA) hat Ende Dezember 2010 die EU-weite Zulassung für Tasigna ${ }^{\circledast}$ (Nilotinib, 2 × 300 mg/ Tag) zur Therapie erwachsener Patienten mit neu diagnostizierter, Philadelphia-Chromosom positiver, chronischer myeloischer Leukämie ( $\mathrm{Ph}+-\mathrm{CML})$ in der chronischen Phase erteilt.}

Die Zulassung basiert auf den 12-Monats-Daten der ENESTndStudie [Saglio G et al. N Engl J Med. 2010; 362:2251-9]. Dass Nilotinib die Patienten signifikant besser vor der Krankheitsprogression schützt als der bisherige Therapiestandard Imatinib wurde auch durch die auf dem ASH (American Society of Hematology)-Meeting im Dezember 2010 von T. P. Hughes mündlich vorgestellte 24-MonatsAuswertung bestätigt. „Bei Anwendung von Nilotinib kommt es bei deutlich weniger Patienten zur Progression der CML in fortgeschrittene Krankheitsphasen. Dies spricht für den frühen Einsatz dieses gezielt entwickelten Tyrosinkinase-Inhibitors, zumal ein verlängertes progressionsfreies
Überleben ein etablierter prädiktiver Parameter für ein besseres Gesamtüberleben ist", erklärte zudem Andreas Hochhaus auf der letztjährigen Jahrestagung der europäischen Hämatologen (EHA, European Hematology Association). „Darüber hinaus zeigte Nilotinib im Rahmen sämtlicher Studienauswertungen eine gute Verträglichkeit - ein essentieller Faktor bei einer chronischen Erkrankung wie CML", sagte er.

In der neu zugelassenen Erstlinienbehandlung der CML werden die 2 x 300 mg Nilotinib in Hartkapseln zu 150 mg verabreicht, wohingegen in der Zweitlinientherapie $(2$ x $400 \mathrm{mg})$ Hartkapseln zu $200 \mathrm{mg}$ zum Einsatz kommen. $\mathbf{d b}$ Nach Informationen von Novartis Pharma

\section{Kurz notiert}

Aktuelle Studienergebnisse auf www.onkodin.de - auch zu Bendamustin Auf der Internetplattform www.onkodin.de beurteilen klinisch erfahrene Onkologen neueste Forschungsergebnisse im Kontext aktueller Behandlungsempfehlungen. So kommentierte Hartmut Link, Kaiserslautern, Ergebnisse von Bendamustin-Studien, die auf dem ASH (American Society of Hematology)-Meeting im Dezember 2010 präsentiert wurden. In der einen wurde die Wirksamkeit von Bendamustin (Ribomustin ${ }^{\oplus}$ ) mit der von Chlorambucil bei Patienten mit chronisch lymphatischer Leukämie verglichen. Hier führte die Bendamustin-Therapie u.a. zu höheren Remissionsraten und einem längeren progressionsfreien Überleben und das bei guter Lebensqualität [Knauf WU et al. Blood (ASH Annual Meeting Abstracts) 2010;116:Abstr. 2449]. Link dazu in seinem Kommentar: „Diese Follow-up-Daten der Primärtherapiestudie (...) bestätigen die Daten der Erstpublikation mit einer deutlich besseren Wirksamkeit von Bendamustin. In der zweiten Arbeit hatte die STiL-Gruppe bei Patienten mit follikulären Lymphomen in der Second-Line die Regime Bendamustin-Rituximab und Fludarabin-Rituximab verglichen [Rummel MJ et al. Blood (ASH Annual Meeting Abstracts) 2010;116:Abstr. 856]. Sein Kommentar zu dieser Studie: „Die sehr gute Wirksamkeit von Bendamustin- im Vergleich zu Fludarabin-Rituximab bei guter Verträglichkeit etabliert diese Kombination bei der Therapie follikulärer Lymphome" $\mathrm{db}$ Nach Information von Mundipharma 